\title{
Status of GEO 600
}

B Willke ${ }^{1}$, P Aufmuth ${ }^{1}$, C Aulbert ${ }^{2}$, S Babak $^{3}$, R Balasubramanian $^{3}$, B W Barr ${ }^{4}$, S Berukoff ${ }^{2}$, G Cagnoli ${ }^{4}$, C A Cantley ${ }^{4}$, M M Casey ${ }^{4}$, S Chelkowski ${ }^{1}$, D Churches ${ }^{3}$, C N Colacino ${ }^{1}$, D R M Crooks ${ }^{4}$, C Cutler $^{2}$, K Danzmann ${ }^{1}$, R Davies ${ }^{3}$, R J Dupuis ${ }^{4}$, E Elliffe ${ }^{4}$, C Fallnich ${ }^{5}$, A Freise ${ }^{1}$, S Goßler ${ }^{1}$, A Grant ${ }^{4}$, H Grote ${ }^{1}$, S Grunewald ${ }^{2}$, J Harms ${ }^{1}$, G Heinzel ${ }^{1}$, I S Heng ${ }^{1}$, A Hepstonstall ${ }^{4}$, M Heurs ${ }^{1}$, M Hewitson ${ }^{4}$, S Hild ${ }^{1}$, J Hough ${ }^{4}$, R Ingley ${ }^{6}$, Y Itoh ${ }^{2}$, O Jennrich ${ }^{4}$, R Jones ${ }^{4}$, S H Hutter ${ }^{4}$, K Kawabe ${ }^{1}$, C Killow ${ }^{4}$, K Kötter ${ }^{1}$, B Krishnan ${ }^{2}$, V Leonhardt ${ }^{1}$, H Lück ${ }^{1}$, B Machenschalk ${ }^{2}$, M Malec ${ }^{1}$, R A Mercer ${ }^{6}$, C Messenger ${ }^{6}, \mathbf{S}$ Mohanty ${ }^{2}$, K Mossavi ${ }^{1}$, S Mukherjee ${ }^{2}$, S Nagano ${ }^{1}$, G P Newton ${ }^{4}$, M A Papa ${ }^{2}$, M Perreur-Lloyd ${ }^{4}$, M Pitkin $^{4}$, M V Plissi ${ }^{4}$, V Quetschke ${ }^{1}, V_{R^{6}}{ }^{6}, \mathbf{S} \operatorname{Reid}^{4}$, L Ribichini ${ }^{1}$, D I Robertson ${ }^{4}$, N A Robertson ${ }^{4}$, S Rowan $^{4}$, A Rüdiger $^{1}$, B S Sathyaprakash ${ }^{3}$, R Schilling ${ }^{1}$, R Schnabel ${ }^{1}$, B F Schutz ${ }^{2,3}$, F Seifert ${ }^{1}$, A M Sintes ${ }^{7}$, J R Smith ${ }^{1}$, P H Sneddon ${ }^{4}$, K A Strain ${ }^{4}$, I Taylor ${ }^{3}$,

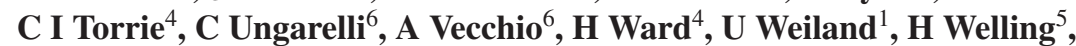
P Williams ${ }^{2}$, W Winkler ${ }^{1}$, G Woan ${ }^{4}$ and I Zawischa ${ }^{5}$

\footnotetext{
${ }^{1}$ Max-Planck-Institut für Gravitationsphysik (Albert-Einstein-Institut) und Universität Hannover, Callinstr. 38, D-30167 Hannover, Germany

${ }^{2}$ Max-Planck-Institut für Gravitationsphysik (Albert-Einstein-Institut), Am Mühlenberg 1, 14476 Golm, Germany

${ }^{3}$ Department of Physics and Astronomy, Cardiff University, PO Box 913, Cardiff CF2 3YB, UK

${ }^{4}$ Department of Physics and Astronomy, University of Glasgow, Glasgow G12 8QQ, UK

${ }^{5}$ Laser Zentrum Hannover e. V., Hollerithallee 8, 30419 Hannover, Germany

${ }^{6}$ School of Physics and Astronomy, The University of Birmingham, Edgbaston, Birmingham B15 2TT, UK

${ }^{7}$ Departament de Fisica, Universitat de les Illes Balears, E-07122 Palma de Mallorca, Spain
}

Received 19 September 2003

Published 3 February 2004

Online at stacks.iop.org/CQG/21/S417 (DOI: 10.1088/0264-9381/21/5/006)

\begin{abstract}
The GEO 600 laser interferometer with $600 \mathrm{~m}$ armlength is currently being commissioned as a part of a worldwide network of gravitational wave detectors. Due to the use of advanced technologies such as signal recycling and multiple pendulum suspensions with a monolithic last stage the anticipated sensitivity of GEO 600 is close to the initial sensitivity of km baseline detectors. This paper describes the status of the detector as of November 2003 with special emphasis on its performance during the first serious data-taking periods and on the experimental challenges encountered during the commissioning of the dual-recycled detector.
\end{abstract}

PACS number: $04.80 . \mathrm{Nn}$ 


\section{Introduction}

An international network of earth-bound laser interferometric gravitational wave detectors is currently in the transition from the commissioning phase to long-term data-taking periods. These detectors will be searching for gravitational waves from a number of different astrophysical sources such as supernovae explosions, non-symmetric pulsars, inspiralling binary systems of neutron stars or black holes and remnants of the big bang. Furthermore, unknown sources may produce gravitational waves of detectable strength. A summary of the current understanding of astrophysical sources for gravitational waves and of predicted event rates is given in [1].

Six laser interferometric gravitational wave detectors will be in operation in the near future: three interferometers of the LIGO project [2] in the USA (two interferometers with $4 \mathrm{~km}$ and one with $2 \mathrm{~km}$ baseline), one detector of the French-Italian VIRGO project [3] in Italy with $3 \mathrm{~km}$ baseline, the TAMA300 detector [4] in Japan with a baseline of $300 \mathrm{~m}$ and the British-German GEO 600 detector with $600 \mathrm{~m}$ armlength in Germany. An $80 \mathrm{~m}$ prototype interferometer with the option to extend it to a large-scale detector is under construction by the ACIGA project in Australia [5].

The GEO 600 detector's design was based on the experience with two prototypes: the $10 \mathrm{~m}$ interferometer at Glasgow University and the $30 \mathrm{~m}$ interferometer at the Max-PlanckInstitut für Quantenoptik in Garching, near Munich. The construction of GEO 600 started in 1995 as a German/British collaboration on a site near Hannover in Germany. To improve the sensitivity to gravitational waves an advanced optical layout including signal recycling and a novel design of suspensions for reducing thermal noise effects were included in the detector design. A detailed description of the detector subsystems can be found in [6].

This paper will concentrate on the performance of the GEO 600 detector during the most recent engineering and science runs and will give a detailed description of the calibration process used. The second part of this paper will place special emphasis on the commissioning of dual recycling and the challenges encountered after the installation of the final optics in GEO 600.

\section{Detector performance during the first engineering and science runs}

GEO 600 is a laser interferometric gravitational wave detector with a baseline of $600 \mathrm{~m}$. An advanced optical layout (see figure 1) including two sequential modecleaners [7], folded optical paths in the interferometer arms, power recycling and signal recycling $[8,9]$ was chosen to increase the optical signal produced by a gravitational wave and to reduce noise sources associated with laser noise, beam-pointing fluctuations and the shot-noise of the detected photocurrent. Novel techniques for the seismic isolation systems [10] were included in the detector design to reduce the displacement noise of the mirrors introduced by ground motion and thermal noise in the mirrors and their suspensions.

To gain experience with the alignment and length control of long baseline cavities, one step in the commissioning was the installation of a $1200 \mathrm{~m}$ long Fabry-Perot cavity [11] formed by one interferometer arm and the power-recycling mirror. To reduce the risk of contaminating or damaging the expensive main interferometer mirrors, lower grade test mirrors suspended in steel wire slings were used for the $1200 \mathrm{~m}$ cavity experiment and for the followings steps, namely the commissioning of the power-recycled Michelson interferometer which started in summer 2001. A first engineering test run (E7) was conducted in January 2002 in coincidence with the LIGO interferometers. 


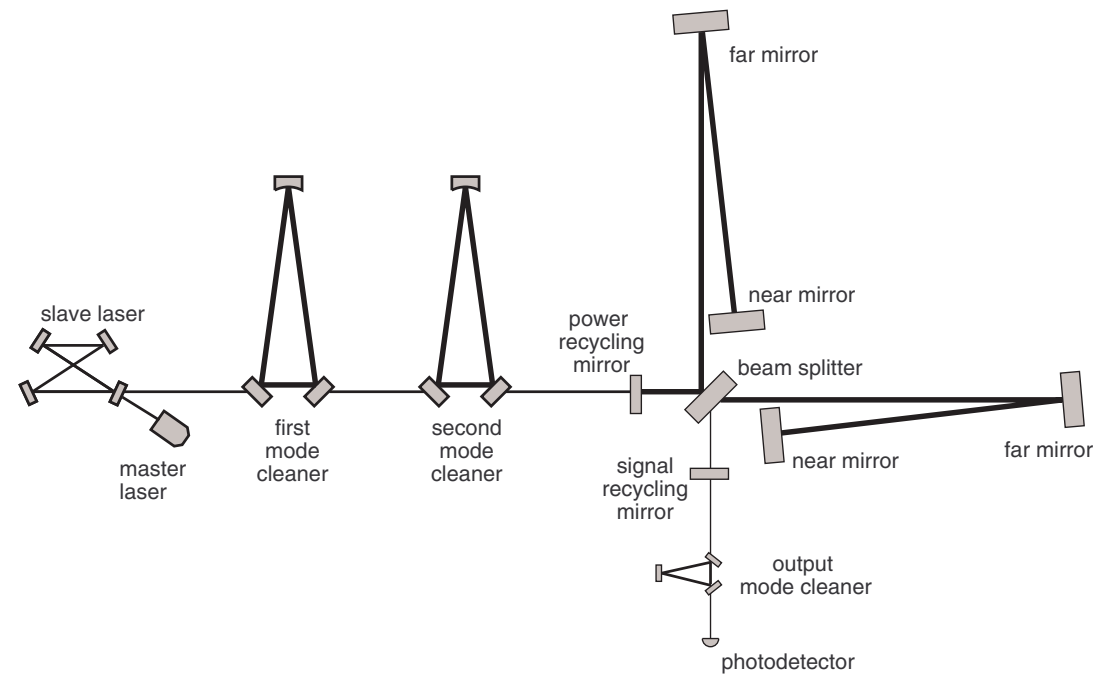

Figure 1. Optical layout of GEO 600: the beam from a $12 \mathrm{~W}$ injection-locked laser system is filtered by two sequential modecleaners and injected into the dual-recycled interferometer. A folded light path is used to increase the round-trip length of the interferometer arms to $2400 \mathrm{~m}$. An output modecleaner will be used to spatially filter the laser mode before it reaches the photodetector.

For this test run the lock acquisition of the laser system, modecleaners and interferometer was automated and the automatic alignment control for the modecleaners [12] was implemented such that no operator interaction with the modecleaner subsystem was needed. The alignment and drift control of the Michelson interferometer was not installed at that time and frequent operator interaction was needed to keep the $600 \mathrm{~m}$ long arms aligned with respect to each other. The main goal of this test run was to try to run the detector for many hours and acquire long stretches of data. These data were used to characterize the behaviour of the detector subsystems, and to provide data with real detector noise on which the data analysis codes could be tested. An overall duty cycle better than $70 \%$ was achieved and the longest uninterrupted operation (longest lock) was for $3 \mathrm{~h}$ and $38 \mathrm{~min}$. The data acquisition system worked reliably and a total of 0.9 terabytes of data was acquired.

Based on the insight gained into the performance of the detector subsystems the E7 test run was followed by an optimization period in which the detector sensitivity was improved substantially (see figure 2). In August and September 2002, the LIGO and GEO 600 laser interferometric gravitational wave detectors were operated in coincidence for 17 days to produce their first data for scientific analysis.

During this so-called S1 run GEO 600 was operating in the power-recycled Michelson interferometer configuration with a reduced power-recycling gain and reduced input laser power. The laser power injected into the first modecleaner was attenuated from $10 \mathrm{~W}$ to $2 \mathrm{~W}$. The overall optical transmission of the modecleaners, phase modulators and isolators was 52\% which left approximately $1 \mathrm{~W}$ of laser light being injected into the interferometer. The power build-up in the power-recycling cavity was 300 which was limited by the $1.3 \%$ transmission of the power-recycling mirror installed during S1. The signal-recycling mirror was not installed and the test mirrors suspended in wire slings were used for the near mirrors of the folded interferometer arms and for the beamsplitter.

Thanks to the automatic alignment system of the modecleaners [12] the longest duration without manual alignment of the modecleaners was more than one year prior to S1 and no 


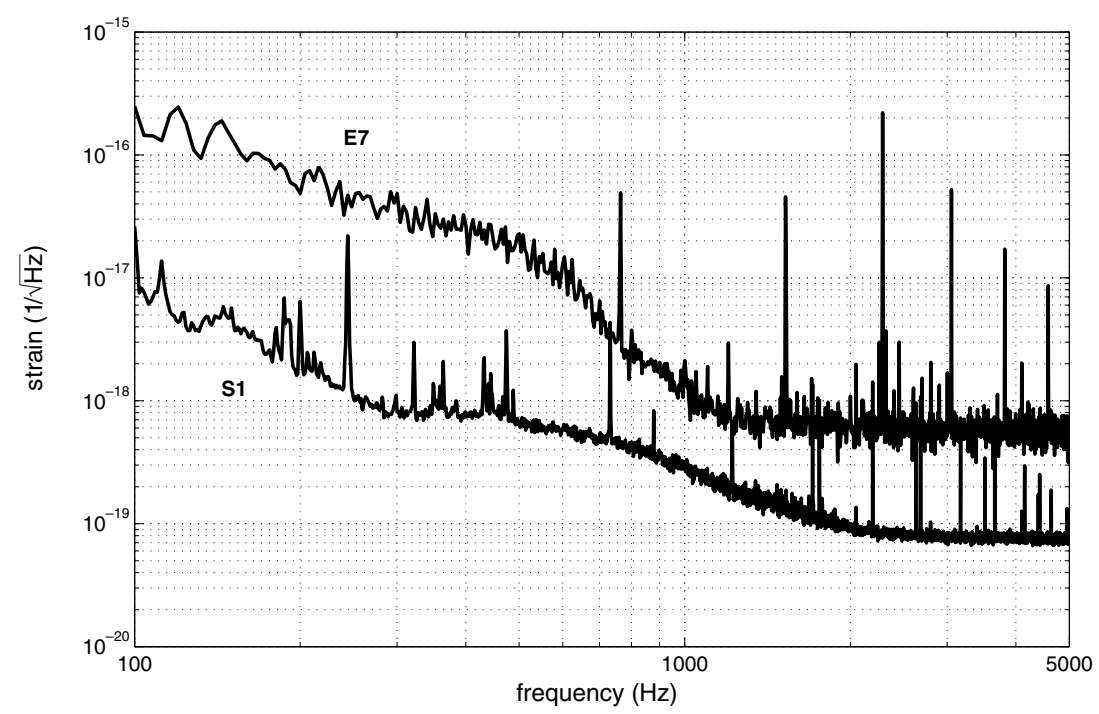

Figure 2. Sensitivity of the GEO 600 detector during an engineering run (E7) in January 2002 and a first science run (S1) in August 2002. The most dominant lines around $244 \mathrm{~Hz}$ and odd harmonics are calibration lines injected into the detector via an electrostatic actuator. The response of the power-recycled Michelson interferometer to these lines allows one to calibrate the detector response and the gains in the relevant control loops.

manual alignment of the modecleaners was undertaken during the S1 run. Manual alignment of the power-recycling cavity and the Michelson interferometer was needed only a few times after major seismic disturbances. An automatic lock-acquisition process was initiated whenever any of the cavities or the Michelson interferometer lost lock. This system and the automatic alignment system for the main interferometer were very stable and reliable. The installation of the automatic alignment system for the Michelson interferometer and for the power-recycling cavity [13] after E7 was a key step towards an S1 duty cycle of more than $98 \%$. The longest continuous $\mathrm{S} 1$ data segment is $128 \mathrm{~h}$.

In the final GEO 600 configuration, radiation pressure from a modulated laser beam which will be aligned onto and reflected by one of the end mirrors will introduce calibration signals with known amplitude into the output signal of the detector. Calibration of GEO 600 during the $\mathrm{S} 1$ run was achieved by imposing known forces on two of the mirrors using electrostatic drives that provided differential control of the interferometer. The spectrum of the applied calibration force consisted of a line at $244 \mathrm{~Hz}$ and its odd harmonics, generated by suitable filtering from a square wave. The signal generator used to produce the series was phase-locked to a GPS stabilized clock with which the data acquisition system was also synchronized. The electrostatic actuators were calibrated with respect to electromagnetic actuators one stage higher up on the same suspension chains. These were in turn calibrated by applying forces large enough to enable simple fringe counting. The actuators were found to be adequately linear to allow this calibration method to succeed with good reliability. The calibrated gravitational wave channel was generated by measuring the amplitudes of the calibration peaks in $1 \mathrm{~s}$ frames of data. These measurements were used to determine the unknown calibration factors (essentially the overall optical transfer function magnitude and the gain of the relevant control loops) by fitting the data to a model based on previously measured transfer functions of the electromechanical control system. The calibration coefficients were smoothed over periods of 
$1 \mathrm{~min}$. Suitable time-domain digital filters were generated to produce a calibrated gravitational wave channel. The overall calibration accuracy was about $4 \%$ for signal frequencies above $200 \mathrm{~Hz}$ and $6 \%$ between $50 \mathrm{~Hz}$ and $200 \mathrm{~Hz}$. Further details of this process can be found in [14].

The S1 science run is an important milestone for LIGO and GEO 600, providing the first data for scientific analysis for two of the newest generation of gravitational wave interferometers. Even though the detectors were operated in a preliminary configuration with many features not implemented, the data were relatively well behaved, and the sensitivity good enough to improve on prior observations with broadband gravitational wave detectors $[15,16]$.

\section{Commissioning of the dual-recycled detector}

The S1 run was followed by the last major installation step towards the final dual-recycled optical configuration of GEO 600. The signal-recycling mirror was installed and the last test optics were replaced by the final mirrors suspended as triple pendulums with a final stage made entirely from fused silica $[17,18]$. Several experimental challenges arose in conjunction with these installations in early 2003.

\subsection{Thermal compensation of radius-of-curvature mismatch}

After the installation of all final mirrors it was found that the interference defect (which is the ratio of the light power at the dark port to the power in the interferometer) was $0.25 \%$ and a sombrero-like beam pattern could be seen at the dark port of the interferometer. This was due to a radius-of-curvature (ROC) mismatch of the far folding mirrors in the interferometer arms (see figure 1). To achieve an optically stable operation of the recycling cavities these mirrors were designed to have an ROC of $640 \mathrm{~m}$ [19]. The installed mirrors however have a ROC of $666 \mathrm{~m}$ and $687 \mathrm{~m}$ for the north and east folding mirrors, respectively.

In addition to the lowered power build-up $(200 \mathrm{~W}$ instead of $300 \mathrm{~W}$ that are to be expected for a perfect interference condition) the length-control system of the Michelson interferometer was unfavourably affected by the ROC mismatch. With the test mirrors and a power-recycling mirror with $1.3 \%$ transmission a small contrast defect could be achieved which leads to a largely overcoupled cavity with steep slopes for the length-control error signal. Due to the high contrast defect with the final mirrors in place the detector became much more sensitive to misalignments which moved the power-recycling cavity closer to the impedance-matched case where the error signal for the Michelson length control vanishes. This effect did not allow a stable operation of the power-recycled Michelson interferometer.

Due to the high costs and long delivery times of new mirrors we could not replace the mirrors. Instead we installed a thermal compensation scheme [20] to correct for the ROC mismatch. We adjust the ROC of the mirrors which are made of fused silica (Suprasil 1 from Heraeus, diameter $18 \mathrm{~cm}$, thickness $10 \mathrm{~cm}$ ) with radiative heating from a ring heater located about $5 \mathrm{~cm}$ behind the east far folding mirror.

The heater is made of a ring-like Duran glass rod wrapped with a $100 \mu \mathrm{m}$ thick stainless steel ribbon that is heated by passing a dc current through it. This heater creates a thermal gradient inside the mirror, and the resulting thermal expansion decreases the ROC of the front surface. At a heating power of $74 \mathrm{~W}$ the mismatch in the ROCs is minimized and the dark port power is decreased to about $20 \mathrm{~mW}$ at an intracavity power close to $300 \mathrm{~W}$. 


\subsection{Lock acquisition of the dual-recycled detector}

After the contrast defect of the Michelson interferometer was reduced, a stable operation of the power-recycled interferometer with misaligned signal-recycling mirror was again possible, and the commissioning of the dual-recycled detector could start. On the basis of the experiences with the $30 \mathrm{~m}$ prototype in Garching [9], we first tried the most simple lock-acquisition scheme possible which is to align the optical system as well as possible and to turn on the different length-control systems at the same time. Experiments confirmed by simulations showed that this lock-acquisition approach does not work for the GEO 600 detector.

Three different optical subsystems need to be considered to describe the lock acquisition of the dual-recycled detector (DRMI): the Michelson interferometer (MI) with its differential and common mode degrees of freedom, the power-recycling cavity (PRC) formed by the power-recycling mirror and the MI at its dark fringe operation point and the signal-recycling cavity (SRC) set up by the signal-recycling mirror and the MI. The MI behaves like a mirror with variable reflectivity which is defined by the tuning of the MI differential degree of freedom. The term tuning is used throughout this paper to indicate the state of the length degree of freedom between the dark and the bright fringes of the MI and between resonance and anti-resonance of the various cavities.

The three control loops for the PRC, the MI and the SRC are strongly coupled: a proper error signal for the MI exists only after the PRC has been locked to the laser light. While in the case of the power-recycled MI (without signal recycling) the PRC lock is stable for almost any tuning of the Michelson interferometer, in the case of dual recycling, there exist combinations of the Michelson and signal-recycling tuning for which the power-recycling lock is not stable. This instability is caused by the modulation sidebands used for the power-recycling lock being resonant within the dual-recycled Michelson. A possible solution for this problem is the approach of initially locking the Michelson (while the PRC is already locked), with the signalrecycling mirror being in a largely detuned position, which avoids the PR control sidebands to become resonant. Using this lock-acquisition scheme (for more details, see [21]) we were able to lock the detector and keep it at a largely detuned dual-recycling operation point for up to $15 \mathrm{~min}$.

Even though this demonstrated the feasibility of our approach, a successful lock acquisition was rare and depended strongly on environmental conditions. More investigations led to the conclusion that alignment fluctuations especially in the tilt degree of freedom, were too large to allow for frequent locks. These alignment fluctuations were traced back to a coupling of longitudinal motion of the suspension point via the triple pendulum suspension into the tilt of the mirrors. A new seismic isolation system based on a feed-forward control from seismometers on the building foundations to piezoelectric actuators in the legs that support the pendulum suspension structure was installed to reduce the motion of the suspension point.

An intermediate step was introduced in the lock-acquisition process which uses the signal of the dark-port photodiode demodulated at twice the MI modulation frequency. This signal is a measure of the power build-up of the MI modulation sidebands and hence of the tuning of the SRC and allows us to lock the DRMI in the detuned state. Once the lock-acquisition process was successful the automatic alignment systems can be switched on and a signal picked of at the AR coating of the MI beamsplitter can be used for the error signal of the SRC lengthcontrol system. An automatic system with look-up tables for the MI and SRC modulation frequencies and demodulation phases gradually tunes the DRMI to its final operation point. With the feed-forward seismic-noise reduction system typical re-lock durations were reduced to several minutes and the automatic tuning system brings the DRMI to its final operation point 
within less than a minute. At an initial used signal-recycling tuning of $1.2 \mathrm{kHz}$ the DRMI is very stable and continuous lock stretches of more than $24 \mathrm{~h}$ could be achieved.

The next steps in the commissioning of the GEO 600 detector will be a noise optimization before attending the S3 data-taking period (November-December 2003) in coincidence with the LIGO detectors. A sensitivity optimization period in conjunction with the installation of the final output optics will follow the S3 run and the injected power will be increased from $2 \mathrm{~W}$ to $10 \mathrm{~W}$. As a last step in the commissioning the power-recycling mirror will be replaced to enhance the circulating power in the DRMI to its design value.

\section{Conclusion}

Since November 2003 GEO 600 is operating as the first long-baseline GW detector in the dual-recycling optical configuration. Several technical problems had to be solved after the installation of the final mirrors and the signal-recycling mirror in late 2002: a thermal compensation scheme was installed to reduce the ROC mismatch of the interferometer mirrors, an active feed-forward system was implemented to reduce the mirror tilt motion and a new locking scheme was invented to allow for fast and reliable lock acquisition. Stable operation with lock stretches of more than $24 \mathrm{~h}$ was demonstrated. As GEO 600 is now in its final optical configuration sensitivity optimization will be the major task for the near future.

\section{Acknowledgments}

The authors would like to thank PPARC in the UK, the BMBF and the state of Lower Saxony in Germany.

\section{References}

[1] Schutz B F 1999 Class. Quantum Grav. 16 A131

[2] Sigg D et al 2004 Class. Quantum Grav. 21 S409

[3] Acernese F et al 2004 Class. Quantum Grav. 21 S385

[4] Takahashi R et al 2004 Class. Quantum Grav. 21 S697

[5] Ju L et al 2004 Class. Quantum Grav. 21 S887

[6] Willke B et al 2001 Class. Quantum Grav. 191377

[7] Goßler S et al 2003 Rev. Sci. Instrum. 743787

[8] Meers B J et al 1988 Phys. Rev. D 382317

[9] Heinzel G et al 1998 Phys. Rev. Lett. 815493

[10] Plissi M V et al 2000 Rev. Sci. Instrum. 712539

[11] Freise A et al 2001 Class. Quantum Grav. 191389

[12] Grote H et al 2001 Class. Quantum Grav. 191849

[13] Grote $\mathrm{H}$ et al 2004 Class. Quantum Grav. 21 S441

[14] Hewitson M et al 2003 Class. Quantum Grav. 20885

[15] Nicholson D et al 1996 Phys. Lett. A 218175

[16] Allen B et al 2004 Class. Quantum Grav. 21 S671

[17] Smith J R et al 2004 Class. Quantum Grav. 21 S1091

[18] Goßler S et al 2004 Class. Quantum Grav. 21 S923

[19] Winkler W et al 2003 in preparation

[20] Lück H et al 2004 Class. Quantum Grav. 21 S985

[21] Grote H et al 2004 Class. Quantum Grav. 21 S473 